\title{
Serotonin stimulates Echinococcus multilocularis larval development
}

\author{
Michaela Herz ${ }^{*}$ (1) and Klaus Brehm*
}

\begin{abstract}
Background: Serotonin is a phylogenetically ancient molecule that is widely distributed in most metazoans, including flatworms. In addition to its role as a neurotransmitter, serotonin acts as a morphogen and regulates developmental processes. Although several studies have focused on the serotonergic nervous system in parasitic flatworms, little is known on the role of serotonin in flatworm development.

Methods: To study the effects of serotonin on proliferation and development of the cestode Echinococcus multilocularis, we cloned the genes encoding the E. multilocularis serotonin transporter (SERT) and tryptophan hydroxylase (TPH), analyzed gene expression by transcriptome analysis and whole mount in situ hybridization (WMISH) and performed cell culture experiments.
\end{abstract}

Results: We first characterized orthologues encoding the SERT and TPH, the rate-limiting enzyme in serotonin biosynthesis. WMISH and transcriptomic analyses indicated that the genes for both SERT and TPH are expressed in the parasite nervous system. Long-term treatment of parasite stem cell cultures with serotonin stimulated development towards the parasite metacestode stage. Mature metacestode vesicles treated with serotonin showed increased rates of incorporation of the thymidine analogue 5-ethynyl-2'-deoxyuridine (EdU), indicating stimulated cell proliferation. In contrast, treatment with the selective serotonin reuptake inhibitor paroxetine strongly affected the viability of parasite cells. Paroxetine also caused structural damage in metacestode vesicles, suggesting that serotonin transport is crucial for the integrity of parasite vesicles.

Conclusions: Our results indicate that serotonin plays an important role in E. multilocularis development and proliferation, providing evidence that the E. multilocularis SERT and TPH are expressed in the nervous system of the protoscolex. Our results further suggest that the E. multilocularis SERT has a secondary role outside the nervous system that is essential for parasite integrity and survival. Since serotonin stimulated E. multilocularis metacestode development and proliferation, serotonin might also contribute to the formation and growth of the parasite in the liver.

Keywords: Cestode, Proliferation, Serotonin, Serotonin transporter, Tryptophan hydroxylase, Nervous system

\section{Background}

The metacestode stage of the cestode Echinococcus multilocularis is the causative agent of alveolar echinococcosis, a severe zoonosis prevalent in the northern hemisphere [1]. Oral uptake of infectious eggs, which harbor the oncosphere, leads to infection of intermediate hosts, such

*Correspondence: michaela.herz@uni-wuerzburg.de; kbrehm@hygiene uni-wuerzburg.de Institut für Hygiene und Mikrobiologie, Universität Würzburg, Josef-Schneider-Straße 2/E1, 97080 Würzburg, Germany as small rodents and humans. The oncosphere hatches from the egg in the small intestine, penetrates the epithelium and gains access to the inner organs, especially the liver, where it transforms into the metacestode larval stage. The metacestode invasively grows inside the liver as a multi-vesicular parasite tissue [2]. In advanced infections of natural intermediate hosts, the metacestode vesicles form brood capsules with protoscoleces, which eventually develop into the adult stage when transmitted to a definite host or "re-differentiate" within the intermediate host towards the metacestode stage [3]. During 
these developmental transitions the nervous system undergoes remarkable changes $[4,5]$.

The morphology of the serotonergic nervous system has been described for several cestodes and trematodes [6-12], including Echinococcus granulosus [4, 13, 14] and $E$. multilocularis [5]. Functional studies have shown that serotonin is required for the maintenance of contractility in muscle fibers isolated from Schistosoma mansoni adults [15], increases motility in S. mansoni sporocysts [16], influences scolex and strobila motility in Hymenolepis diminuta [17] and increases motility of E. granulosus protoscoleces [4] and Mesocestoides corti tetrathyridia [18].

In addition to its role as a neurotransmitter, serotonin is also known to act as a morphogenic factor that influences developmental processes [19]. Depending on the context, serotonin can induce cell proliferation, differentiation or apoptosis [20-26]. Serotonin also plays an important role during traumatic regeneration of the planarian Polycelis tenuis [27] and influences miracidial transformation of $S$. mansoni (transition from free-living to parasitic stage) $[28,29]$. However, little is known about the developmental role of serotonin in cestodes. In a previous study we and our colleagues showed that exogenously supplied serotonin induces re-differentiation of $E$. granulosus protoscoleces towards the metacestode stage [4]. Whether this is also the case for E. multilocularis has not been investigated to date. Furthermore, the influence of serotonin on other larval transitions, such as the development of the oncosphere towards the metacestode or the proliferation of metacestode vesicles, has not yet been addressed.

The developmental transitions of E. multilocularis larvae within the intermediate host can be mimicked in vitro using culture systems for metacestode vesicles and parasite stem cells that we have previously developed [30-32]. In the study reported here, we investigated the effect of serotonin on E. multilocularis proliferation and larval development. The results show that serotonin significantly induced proliferation in metacestode vesicles and stimulated vesicle development in primary (stem) cell cultures. We also were able to demonstrate that inhibition of serotonin transport with the selective serotonin reuptake inhibitor (SSRI) paroxetine affected both the structural integrity of metacestode vesicles and the viability of primary parasite cells.

\section{Methods}

\section{Parasite material}

Parasite material was isolated from Mongolian jirds (Meriones unguiculatus) where it was maintained through serial peritoneal passages, as described previously [31, 33]. Parasite axenic (host cell-free) primary stem cell cultures were set up from in vitro-cultivated metacestode vesicles essentially as previously described $[31,33]$. Protoscoleces were obtained from parasite material freshly isolated from Mongolian jirds, as described by Brehm et al. [34] with slight modifications. Briefly, parasite material in phosphate buffered saline (PBS) was shaken vigorously for $10 \mathrm{~min}$ to free protoscoleces from metacestode tissue to which they were attached. The suspension was first filtered through a $150-\mu \mathrm{m}$ gauze to remove debris, then filtered through a $30-\mu \mathrm{m}$ gauze filter that retained the protoscoleces. Protoscoleces were collected, resuspended in PBS and transferred to a Petri dish. Slow rotation of the dish separated out calcium bodies, which settled at the bottom, and the protoscoleces, which accumulated in the middle. Protoscoleces were then collected and activated by incubation in $0.05 \%$ w/v pepsin (pH 2 in Dulbecco's Modified Eagle Medium (DMEM) for $30 \mathrm{~min}$ at $37{ }^{\circ} \mathrm{C}$ and $125 \mathrm{rpm}$ (modified from Fernández et al. [35]). After washing three times with PBS, protoscoleces were incubated in $0.2 \% \mathrm{w} / \mathrm{v}$ sodium taurocholate ( $\mathrm{pH} 7.4$ in DMEM) for $3 \mathrm{~h}$ at $37^{\circ} \mathrm{C}$ and $125 \mathrm{rpm}$. Finally, protoscoleces were washed in PBS and fixed in $4 \%$ paraformaldehyde ( $\mathrm{w} / \mathrm{v}$ ) overnight for use in whole mount in situ hybridizations (WMISH).

\section{Cloning and sequencing}

For cloning and sequencing of the E. multilocularis serotonin transporter (SERT) cDNA (sert) and E. multilocularis tryptophan hydroxylase (TPH) cDNA (tph), primers were designed based on available genomic and predicted coding sequences (CDS) (EmuJ_000391300 and EmuJ_000069500, respectively; downloaded from GeneDB) [36-38]. Partially overlapping fragments were amplified from cDNA libraries [39] using different primer combinations (see Additional file 1: Table S1). Rapid amplification of cDNA ends (RACE) was performed for E. multilocularis tph using the plasmid primer IG4-5' SPR2 (binding to the pJG4-5 plasmid) and an E. multilocularis tph-specific primer with cDNA libraries [39] as template. PCR products were cloned into pJet1.2 using the CloneJET ${ }^{\mathrm{TM}}$ PCR Cloning Kit (Fermentas, St. LeonRot, Germany) and sequenced. Sequences of overlapping fragments were assembled in BioEdit 7.2.5 [40], and the assembled sequences were deposited at the EMBL Nucleotide Sequence Database under the accession numbers LT934126.1 (E. multilocularis sert) and LT934127.1 ( $E$. multilocularis tph).

\section{RNA isolation and reverse transcription}

RNA isolation was performed with the Direct-zol $^{\text {TM }}$ RNA MiniPrep kit (Zymo Research, Freiburg, Germany) according the manufacturer's instructions 
(including DNase treatment). For reverse transcription, the SuperScript ${ }^{\circledR} I V$ Reverse Transcriptase (Invitrogen, Darmstadt, Germany) was used with an Oligo-dT primer (5'-ATC TCT TGA AAG GAT CCT GCA GGA CTT ${ }_{22} \mathrm{VX}-3^{\prime}$ ) according to the manufacturer's instructions.

\section{Quantitative reverse transcription-PCR}

Quantitative reverse transcription (RT-PCR) was performed on the StepOnePlus Real-Time PCR System (Thermo Fisher Scientific, Schwerte, Germany) using the following primers: $5^{\prime}$-CTC CTT CAA AGA GCG TTT G-3' and $5^{\prime}$-TTC GGA CTG TGT ATC G-3' for $E$. multilocularis sert; $5^{\prime}$-ATC AAC TCT GGA TGT GGT$3^{\prime}$ and $5^{\prime}$-GAG GTT AAA TGA TGC GGT GC-3' for $E$. multilocularis tph; and $5^{\prime}$-TGA TGA AAG TGA AGC CAA GGA ACT TGA G- $3^{\prime}$ and $5^{\prime}$-TTC GTC TGG AGC GTC TCA TTC TTA GAG-5') for the reference gene $E$. multilocularis elp. The reaction mixture contained $2 \mu \mathrm{l}$ of 1:5 diluted cDNA, $300 \mathrm{nM}$ each primer ( $200 \mathrm{nM}$ for $E$. multilocularis sert) and the HOT FIREPol ${ }^{\circledR}$ EvaGreen ${ }^{\circledR}$ qPCR Mix (ROX) (Solis Biodyne, Düsseldorf, Germany). The following cycling program was used: $95^{\circ} \mathrm{C}, 15 \mathrm{~min}$; then $95{ }^{\circ} \mathrm{C} / 15 \mathrm{~s}, 60{ }^{\circ} \mathrm{C} / 20 \mathrm{~s}\left(58{ }^{\circ} \mathrm{C} / 20 \mathrm{~s}\right.$ for E. multilocularis sert) for 40 cycles; and a final extension at $72^{\circ} \mathrm{C}$ for $20 \mathrm{~s}$; fluorescence was measured at $72{ }^{\circ} \mathrm{C}$. The amplification product specificity was assessed by melting curve analysis. The experiment was carried out with three technical and three biological replicates. The amplification efficiency was calculated with linREG [41, 42], and the relative gene expression was calculated with the formula of Pfaffl [43]. Statistical analysis was performed in fgStatistics [44] using a permutation test with 5000 pairwise resampling cycles.

\section{Bioinformatic analyses}

cDNA sequences for E. multilocularis sert (LT934126.1) and E. multilocularis tph (LT934127.1) were used for BLASTN searches (E-value $<1 \mathrm{e}-10$, identities $>95 \%$, coverage $>3 \%$ ) against the $E$. multilocularis genome at WormBaseParaSite database (WBPS 9) [36, 45-47] to determine exon/intron boundaries. For domain analyses, cDNA sequences were translated into protein sequences with BioEdit 7.2.5 six-frame translations [40] which then were searched with SMART 8.0 for Pfam domains $[48,49]$. To investigate conservation, BLASTP searches (E-value $<1 \mathrm{e}-10$, identities $>50 \%$, coverage $>90 \%$ ) with the sequences of the active domains were performed against the non-redundant protein sequences of Homo sapiens (taxid: 9606) and Schistosoma mansoni (taxid: 6183) in the National Center for Biotechnology Information (NCBI) database. A multiple sequence alignment for the sodium neurotransmitter symporter family (SNF) domains of serotonin transporters from different organisms was generated in BioEdit 7.2.5 (40) using MUSCLE v3.8.31 (4 iterations) $[50,51]$.

\section{Transcriptome data analysis}

Available RNA-Seq data [36] (ENA sample accessions: ERS094035, ERS094036, ERS094037, ERS094038, ERS094039, ERS016464, ERS018054, ERS018053) was mapped to the new version of the E. multilocularis genome downloaded from WormBaseParaSite WBPS7 [36, 45, 46, 52] using Hisat2 v2.0.5 [53]. Reads per transcript (annotations downloaded from WormBaseParaSite WBPS7) were counted with HTSeqCount v0.7.1 [54], using a minimum quality score of 30 to filter out low quality or multiple mapped reads. TPM (Transcripts Per kilobase of exon per Million transcripts mapped) values were calculated for all transcripts.

\section{Whole-mount in situ hybridization}

For the synthesis of digoxigenin-labeled probes, cDNA fragments were amplified using the primers SERT_A_dw (5'-GAA TGC TGT AGA TGT GGT TAT GG-3') and SERT2_up (5'-CTG GTC CCA CAG TTG ATT GC-3') for E. multilocularis sert, and IG 4-5-SPR2 (5'-CTT ATG ATG TGC CAG ATT ATG-3') and TPH_2_up (5'-CAG AGA GGC GAT ACC CAA CTC-3') for E. multilocularis tph. PCR products were cloned into pJet1.2 using CloneJET $^{\mathrm{TM}}$ PCR Cloning Kit (Fermentas, St. Leon-Rot, Germany) and amplified with the primers T7 Plus 2 (5'AGA AGA GTA ATA CGA CTC ACT ATA GG-3') and 5-SP6+pJET1.2.Rev-3 (5'-ATA ATT TAG GTG ACA CTA TAG AAC ATC GAT TTT CCA TGG CAG-3'). Digoxigenin-labeled probes were synthesized by in vitro transcription with T7 and SP6 polymerases (New England Biolabs, Frankfurt/Main, Germany) according to the manufacturer's instructions. WMISH for protoscoleces and metacestodes was performed as described previously [55] using the sense probe as control. Images of metacestodes and Z-stack images of protoscoleces were generated by confocal microscopy (Leica TCS SP5; Leica Microsystems, Wetzlar, Germany). All images were processed with Fiji/ImageJ 2.0.0 [56, 57]. A total of 18 protoscoleces from two different isolates (DPZ and MS1010) were analyzed for each gene. The micrographs shown in the Results section are Z-projections (maximum intensity) of 25 (E. multilocularis sert) and 19 (E. multilocularis tph) focal planes. The region of the protoscolex was cut out, converted to RGB values, made into a montage and flattened with the overlay.

\section{Chemicals}

All compounds were purchased from Sigma-Aldrich (Munchen, Germany). The following stock solutions were prepared: $50 \mathrm{mM}$ serotonin hydrochloride in $0.1 \mathrm{M}$ 
hydrochloric acid; $50 \mathrm{mM}$ paroxetine maleate salt in ethanol; and $4 \mathrm{mM}$ 4-chloro-DL-phenylalanine in $\mathrm{H}_{2} \mathrm{O}$. Solutions were filtrated through the Filtropur $\mathrm{S} 0.2$ syringe filter system (Sarsteadt, Nuembrecht, Germany) and stored at $-20{ }^{\circ} \mathrm{C}$ prior to use.

\section{Serotonin treatment of primary cells}

Echinococcus multilocularis primary cells were cultured in 96-well culture plates (Sarsteadt, Nuembrecht, Germany) with $200 \mu \mathrm{l}$ conditioned medium (c-DMEM-A and C-DMEM-B 1:1, described in [33]) under a nitrogen atmosphere. Medium was supplemented with different concentrations of serotonin $(1,10$ or $100 \mu \mathrm{M})$. The number of fully developed metacestode vesicles was determined by light microscopy after 2 weeks of cultivation. Three independent experiments with each three replicates were performed. The number of developed metacestode vesicles was normalized to the control of each experiment. Normalized data were statistically analyzed using GraphPad Prism 7.00 for Windows (GraphPad Software, La Jolla, CA, USA), using a Kruskal-Wallis test with a Dunn's multiple comparisons test to compare all concentrations with the control.

\section{Labeling and detection of 5-ethynyl-2'-deoxyuridine}

Metacestode vesicles with a diameter of 3-4 mm were cultured in a 12-well culture plate with $2 \mathrm{ml}$ c-DMEMA [33] supplemented with serotonin $(1,10$ or $100 \mu \mathrm{M})$ for 7 days. Short-term labeling and subsequent wholemount detection of the thymidine analogue 5-ethynyl2'-deoxyuridine (EdU; Life Technologies, Darmstadt, Germany) were performed as described previously [55]. Samples were analyzed by confocal microscopy (Leica TCS SP5; Leica Microsystems, Wetzlar, Germany). For quantification of EdU-positive cells, two random fields per vesicle were captured, four vesicles per replicate, three replicates per concentration. Cells were identified based on DAPI (4',6-diamidino-2-phenylindole) staining using a custom script in Fiji/ImageJ 2.0.0 [56, 57]. EdU-positive cells were counted manually. The mean percentage of EdU-positive cells was calculated for each replicate. Statistical analysis was performed with GraphPad Prism 7.00 for Windows (GraphPad Software, La Jolla, CA, USA) using an ordinary one-way analysis of variance (ANOVA) with a Dunnett's multiple comparisons test comparing all concentrations with the control.

\section{Inhibitor treatment of metacestode vesicles}

Metacestode vesicles were cultured in a 12-well-plate with $2 \mathrm{ml} \mathrm{c-DMEM-A} \mathrm{[33]} \mathrm{supplemented} \mathrm{with} \mathrm{the} \mathrm{SSRI}$ paroxetine $(1,10$ or $100 \mu \mathrm{M})$ or 4-chloro-DL-phenylalanine $(1,10$ or $100 \mu \mathrm{M})$. The structural integrity of metacestode vesicles was determined by light microscopy and the number of damaged vesicles was counted. Experiments were performed with three biological replicates. The percentages of structurally affected metacestode vesicles were used for statistical analysis with GraphPad Prism 7.00 for Windows (GraphPad Software) using a two-way, repeated measurements (for the time factor) ANOVA with a Dunnett's multiple comparisons test for each time point, comparing all concentrations with the control.

\section{Resazurin assay}

Primary cells were cultured in 96-well plates (Sarsteadt, Nuembrecht, Germany), with $100 \mu \mathrm{l}$ c-DMEM-A [33] supplemented with paroxetine $(1,10$ or $100 \mu \mathrm{M})$ for 2 days. As cytotoxic control, 1\% triton X-100 (SigmaAldrich) was used. A 100- $\mu \mathrm{l}$ aliquot of resazurin (SigmaAldrich) diluted in PBS was added to all wells (final concentration $10 \mu \mathrm{l} / \mathrm{ml}$ ). After $3 \mathrm{~h}$ of incubation, fluorescence was measured at $540 \mathrm{~nm}$ (reference $595 \mathrm{~nm}$ ) with a TECAN ELISA reader (Tecan Group, Crailsheim, Germany). Three independent experiments, each each three technical replicates, were carried out. Statistical analysis was performed with GraphPad Prism 7.00 for Windows (GraphPad Software) using an ordinary one-way ANOVA with a Dunnett's multiple comparisons test to compare all samples with the negative control.

\section{Paroxetine treatment of primary cells for quantitative reverse transciption-PCR}

Primary cells were cultured in 96-well plates (Sarsteadt) with $200 \mu \mathrm{l}$ conditioned medium (c-DMEM-A and c-DMEM-B 1:1, described in [33]) under anaerobic conditions and treated with $10 \mu \mathrm{M}$ paroxetine for 2 days. The cells were then resuspended and transferred to $1.5-\mathrm{ml}$ tubes. After centrifugation at $600 \mathrm{~g}$ for $5 \mathrm{~min}$, the supernatant was discarded and the cells were resuspended in $500 \mu \mathrm{M}$ Trizol $^{\circledR}$ Reagent (Invitrogen) and stored at $-80{ }^{\circ} \mathrm{C}$ until RNA isolation. The experiment was performed with three biological replicates.

\section{Results}

The E. multilocularis serotonin transporter and tryptophan hydroxylase show high homologies to their human homologues within the active domains

Previous in silico analyses showed that a canonical serotonergic pathway, with exception of the monoamine oxidase and serotonin receptor 3, is encoded in both the E. granulosus and E. multilocularis genomes. In the present study, we cloned and sequenced the E. multilocularis genes encoding the serotonin transporter $(E$. multilocularis sert: LT934126.1) and tryptophan hydroxylase (E. multilocularis tph: LT934127.1). E. multilocularis sert comprised 13 exons and 12 introns, localized on 
chromosome 9, and encoded a protein, E. multilocularis SERT, of 640 amino acids. Domain analysis with SMART 8.0 showed that E. multilocularis SERT contained a SNF domain (PF00209) with 12 transmembrane domains, demonstrating the typical domain structure of a neurotransmitter transporter with sodium symporter activity. The E. multilocularis SERT SNF domain showed high homologies to the Schistosoma mansoni (82\% amino acid similarities) and the human (69\% amino acid similarities) SERT SNF domains. Analysis of five paroxetine binding sites of mammalian SERTs [58-61] showed conservation of three residues in E. multilocularis SERT (see Fig. 1).

Using 5'-RACE-PCR we obtained a sequence for $E$. multilocularis tph. Transcriptome information [36] viewed with the Integrative Genomics Viewer [62, 63] suggests that the sequence did not represent the full transcript and was missing the $5^{\prime}$-end with the start codon (see Additional file 2: Figure S1). The partial sequence of E. multilocularis tph comprised 12 exons and 11 introns, localized on chromosome 3 , and encoded a partial

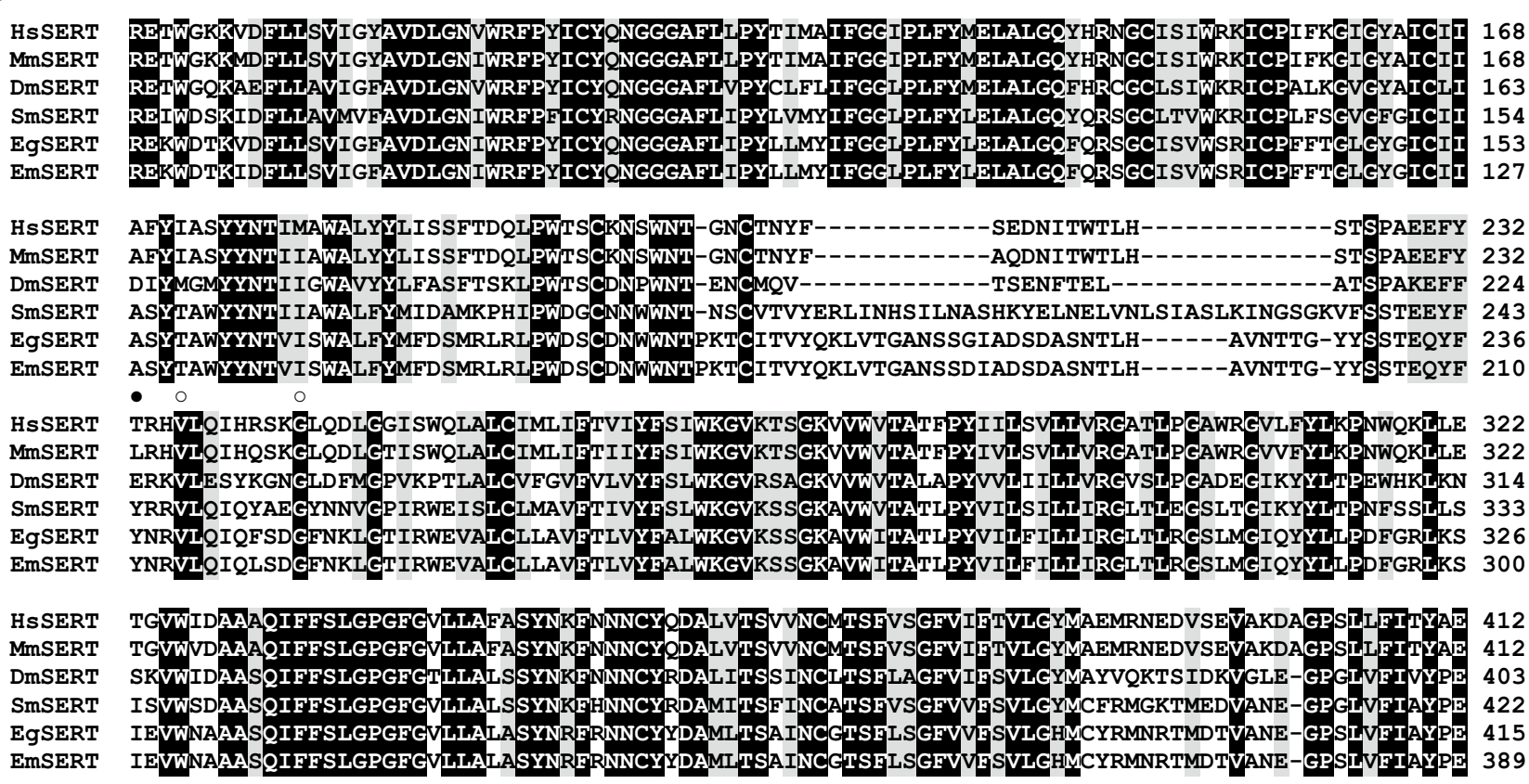

HSSERT AIANMPASTFFAIIFFLMLITLGLDSTFAGLEGVITAVLDEFPHVWAKRRERFVLAVVITCFFGSTVTLTFGGAYVVKLLEEYATGPAVI 502 MMSERT AIANMPASTFFAI I FFLMLITLGLDSTFAGLEGVITAVLDEFPHIWAKRREWFVLIVVITCILGSLLTLTSGGAYVVTLLEEYATGPAVI 502 DMSERT AIATMSGSVFWSI IFFLMLITLGLDSTFGGLEAMITALCDEYPRVIGRRRELFVLLLLAFIFLCALPTMTYGGVVLVNFLNVYGPGLAIL 493 SMSERT AIATLAGSTERAIIFMLMLITLGLDSTFGGLEAIITAIMDSVPAL-SGRREVFVLAVVIYCFIGALPSTTCGGYLVLTMLDRHGAPISIL 511 EgSERT AIATLPGSTFWAI I MMLMLITLGLDSTFGGLEAIITALLDRWPKL-RKRREIVVLIMI IYCYVGALPTTTNGGYY ILTLFDTYGAPFSIL 504 EMSERT AIATLPGSTEWAI IFMLMLITLGLDSTEGGLEAIITALLDRWPKL-RKRREIVVLIMIIYCYVGALPTTTNGGYYILTLFDTYGAPFSII 478

HSSERT MmSERT DISERT SmSERT EgSERT EmSERT

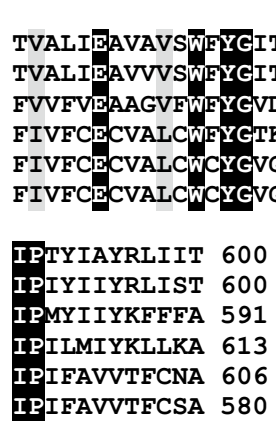

$\begin{array}{lll}\text { HSSERT } & \text { IPTYIAYRLIIT } & 600 \\ \text { MmSERT } & \text { IPIYIIYRLIST } & 600 \\ \text { DMSERT } & \text { IPMYIIYKFFFA } & 591 \\ \text { SmSERT } & \text { IPILMIYKLLKA } & 613 \\ \text { EgSERT } & \text { IPIFAVVTFCNA } & 606 \\ \text { EmSERT } & \text { IPIFAVVTFCSA } & 580\end{array}$

Fig. 1 Comparison of the SNF domain of serotonin transporters (SERT). The multiple sequence alignment utility MUSCLE 3.8.31 [50, 51] was used to align amino acid sequences from Homo sapiens (HSSERT, NP_001036.1), Mus musculus (MmSERT, AAB67172.1), Drosophila melanogaster (DmSERT, NP_523846.2), Schistosoma mansoni (SmSERT, EF061308), Echinococcus granulosus (EgSERT, EUB59773.1) and Echinococcus multilocularis (EmSERT, LT934126.1) SERT. Highly conserved residues are printed white on black background, biochemically similar residues are printed black on gray background. Conserved binding sites for paroxetine [58-61] in E. multilocularis SERT are indicated by black circles; white ones identify substitutions 
protein of 445 amino acids. SMART [48, 49] analysis revealed that the partial E. multilocularis TPH protein contained a complete biopterin-dependent aromatic amino acid hydroxylase (Biopterin_H) domain (PF00351) that is characteristic of TPH and other aromatic amino acid hydroxylases. Within the Biopterin_H domain, E. multilocularis TPH showed high homologies to the $S$. mansoni TPH (82\% amino acid similarities) and to the human TPH2 ( $81 \%$ amino acid similarities), which is predominantly expressed in the brain stem [64].

\section{Expression pattern of $E$. multilocularis sert and $E$.} multilocularis tph indicates expression in the nervous system of the protoscolex

The expression of both E. multilocularis sert and E. multilocularis tph was analyzed in the available transcriptome data sets [36]. While E. multilocularis sert was generally more highly expressed than $E$. multilocularis tph, their expression profiles were similar. In comparison to the other analyzed life-cycle stages, both E. multilocularis sert and tph showed high expression in non-activated and activated protoscoleces and low expression in the metacestode (see Fig. 2).

It should be noted that a serotonergic nervous system has been described for protoscoleces of $E$. multilocularis and $E$. granulosus $[4,5,13,14]$, while no serotonergic nerve cells have been reported in in metacestode vesicles $[4,5]$.
To determine the expression patterns of E. multilocularis sert and E. multilocularis tph in protoscoleces, we performed WMISH using an established protocol [55]. Both the positions of E. multilocularis sert- and E. multilocularis tph-positive cells were relatively constant and corresponded to the location of the serotonergic nervous system in protoscoleces, as previously described [4, 5] (see Fig. 3). Echinococcus multilocularis sert- and E. multilocularis tph-positive cells, respectively, were positioned in the regions of the rostellar ring, the lateral ganglion, the posterior lateral ganglion and the lateral nerve cords. In a few cases, cells were also located in the region of the anterior ring commissure and the medial nerve cords. Rarely did we observe E. multilocularis sert- or E. multilocularis tph-positive cells in the region of the posterior ring commissure. In WMISH experiments for $E$. multilocularis sert or tph on metacestode vesicles, we did not obtain signals for either gene, which might be due to low expression in the metacestode stage.

Taken together, the WMISH and the transcriptome analysis suggest that $E$. multilocularis sert and $E$. multilocularis tph are expressed in the nervous system of the protoscolex.

\section{Serotonin stimulates E. multilocularis larval development in vitro}

To study the influence of serotonin on parasite development, serotonin was applied exogenously to primary cell cultures and metacestode vesicles. Primary cells isolated from metacestode vesicles contain a high percentage of

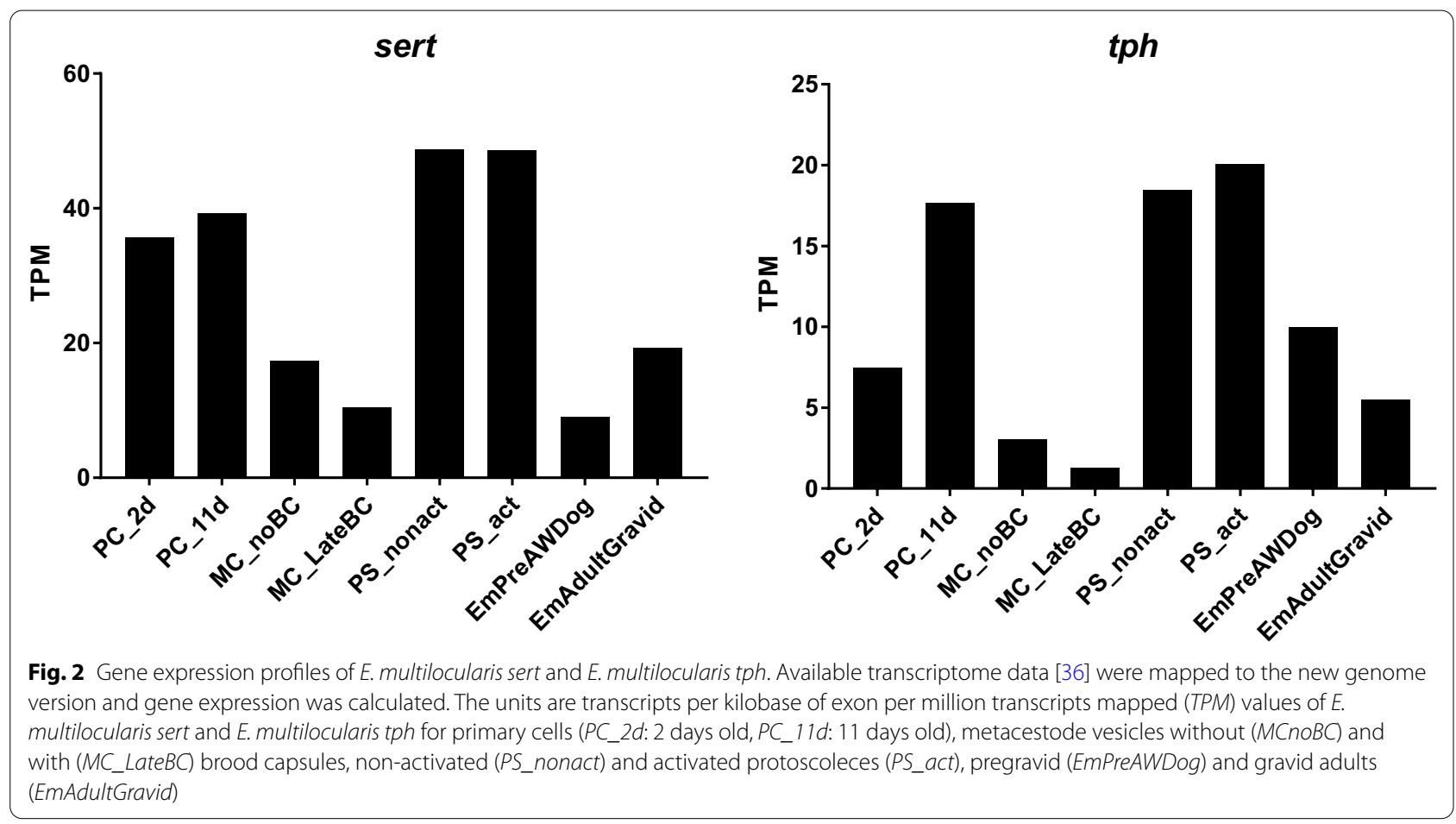



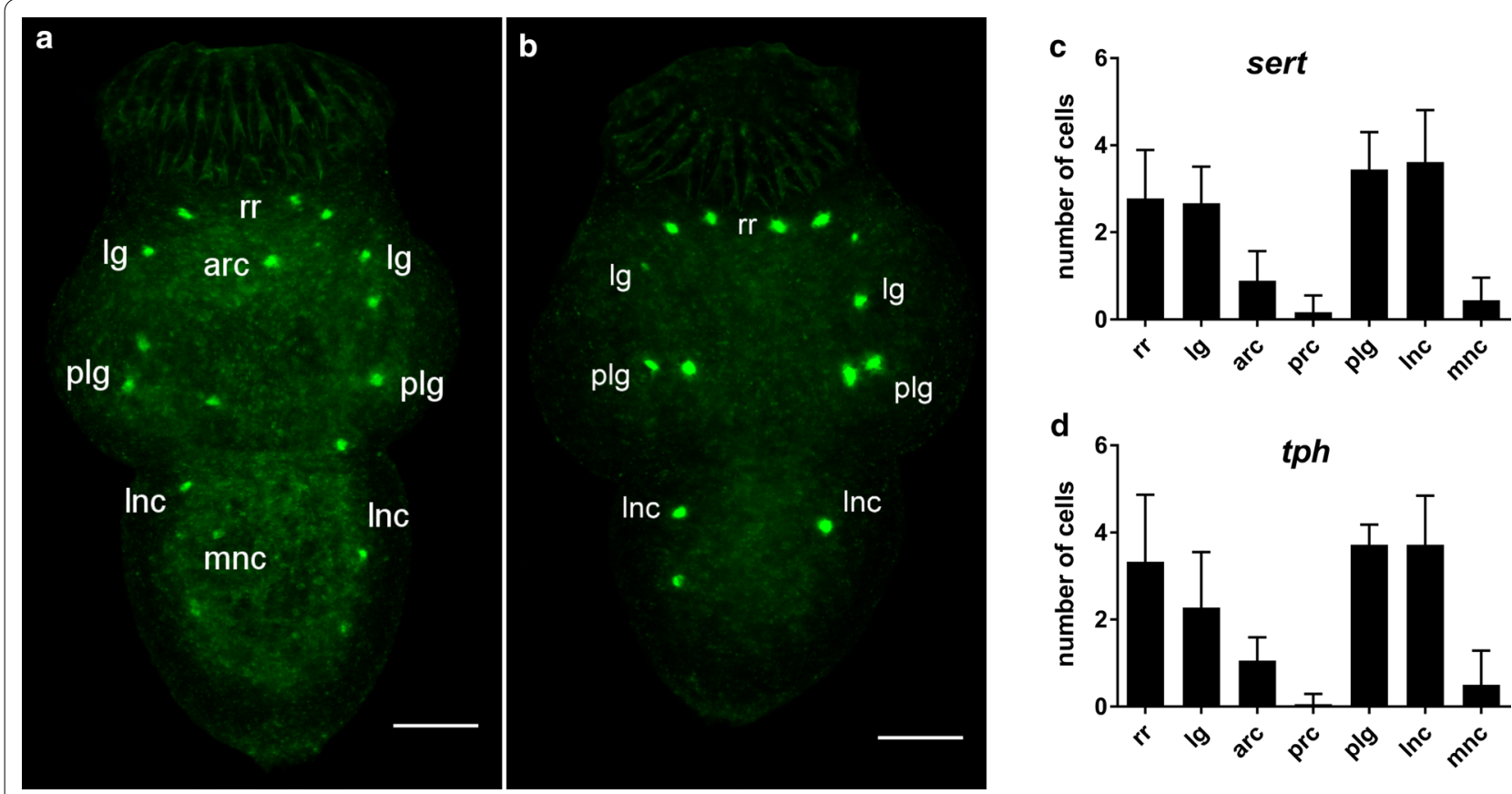

Fig. 3 Expression of E. multilocularis sert and E. multilocularis tph in protoscoleces. a Whole mount in situ hybridization (WMISH) of E. multilocularis sert, $\mathbf{b}$ WMISH of E. multilocularis tph, $\mathbf{c}$ distribution of E. multilocularis sert-positive cells, $\mathbf{d}$ distribution of E. multilocularis tph-positive cells. Echinococcus multilocularis sert- and E. multilocularis tph-positive cells, respectively, were located in the rostellar ring (rr), the lateral ganglion (Ig), the region of the anterior ring commissure (arc), the posterior lateral ganglion ( $p / g)$, the lateral nerve cords (Inc), the medial nerve cords ( $m n n c)$ and rarely in the posterior ring commissure (prc). a, b Micrographs are Z-projections (maximum intensity) of several focal planes. Bars: $25 \mu \mathrm{M}$. c, d Mean number of cells per protoscolex at each position ( $n=18$, isolates DPZ and MS1010). Error bars represent the standard deviation (SD)

pluripotent stem cells [55] and, after the formation of aggregates, develop into metacestode vesicles [31]. This process mimicks the transition from the oncosphere to the metacestode during the early stage of parasite infection in the intermediate host [31, 65]. Exogenous serotonin stimulated the formation of metacestode vesicles from primary cells in a dose-dependent manner. After 14 days, significantly $(P=0.02)$ more metacestode vesicles were detected in samples treated with $100 \mu \mathrm{M}$ serotonin compared to controls (see Fig. 4a). As an indicator for proliferation in metacestodes, we analyzed EdU incorporation and observed that the treatment of mature metacestode vesicles with serotonin stimulated the incorporation of EdU in a dose-dependent manner. Incubation with $100 \mu \mathrm{M}$ serotonin for 7 days significantly $(P=0.003)$ increased the number of EdU-positive cells (see Fig. 4b), indicating proliferation of germinative cells.

In summary, exogenous serotonin stimulates EdU incorporation in metacestodes and vesicle formation in primary cells in E. multilocularis.

\section{Paroxetine affects parasite integrity and cell viability}

To further study the role of serotonin in E. multilocularis, we applied the SSRI paroxetine to parasite cultures. Paroxetine affected the structural integrity of metacestode vesicles. As early as 3 days after exposure, cultures incubated with $100 \mu \mathrm{M}$ paroxetine contained significantly $(P=0.006)$ more collapsed vesicles than untreated control cultures. After 10 days also metacestode cultures treated with $10 \mu \mathrm{M}$ paroxetine showed significantly $(P=0.03)$ more damaged vesicles than control cultures. In contrast, metacestodes incubated with $1 \mu \mathrm{M}$ paroxetine and untreated metacestodes remained intact (see Fig. 5a). On day 14 of the experiment, cultures incubated with $10 \mu \mathrm{M}$ paroxetine contained $29 \%$ collapsed metacestodes, with cultures treated with $100 \mu \mathrm{M}$ paroxetine even reaching $75 \%$ collapsed metacestodes.

To investigate if paroxetine affects cell viability, we performed a resazurin assay on primary cells. Treatment with $100 \mu \mathrm{M}$ paroxetine for 2 days strongly reduced cell viability compared to untreated controls $(P=0.002)$. Incubation with $10 \mu \mathrm{M}$ paroxetine also affected cell viability, although to a lesser extent, and not in a statistically significant manner. Paroxetine at the concentration of $1 \mu \mathrm{M}$ had no effect on primary cells.

To address the question if SERT inhibition by paroxetine is accompanied by a change in expression of $E$. multilocularis sert and tph, we performed quantitative RT-PCR on cDNA from primary cells. Treatment with $10 \mu \mathrm{M}$ paroxetine for 2 days did not significantly alter 

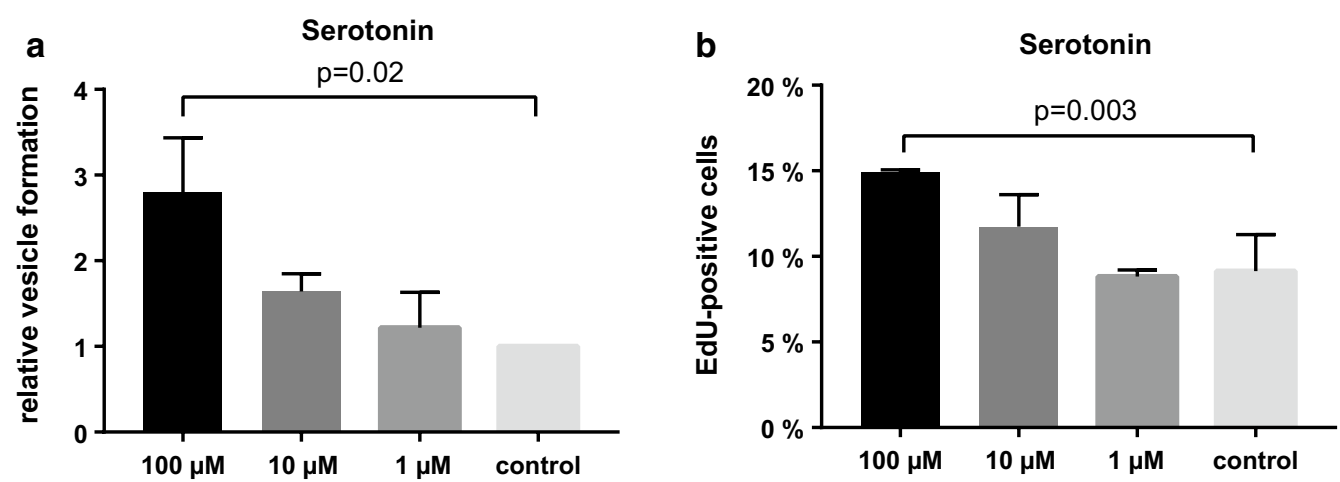

Fig. 4 Effect of serotonin on E. multilocularis. a Metacestode vesicle development from primary cells in the presence of serotonin. Shown is relative vesicle formation compared to untreated control cultures. Error bars represent the SD. $\mathbf{b}$ Proliferation of metacestode vesicles treated with serotonin for 7 days. Shown are the percentages of thymidine analogue 5-ethynyl-2'-deoxyuridine (EdU)-positive cells. Error bars represent the SD
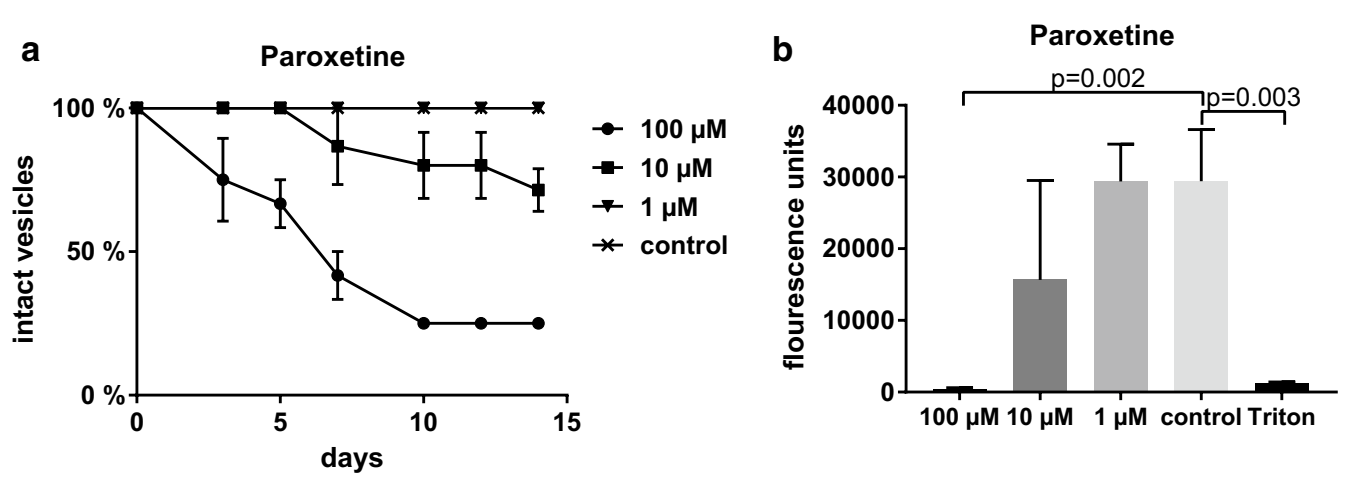

Fig. 5 Effect of paroxetine on E. multilocularis. a Time course of structural integrity of metacestode vesicles in the presence of the selective serotonin reuptake inhibitor paroxetine. Shown are the percentages of intact vesicles. Error bars represent the SD. $\mathbf{b}$ Viability of primary cells after 2 days of treatment with the concentrations of paroxetine indicated. $1 \%$ triton X-100 was used as the cytotoxic control. Shown are fluorescence values. Error bars represent the SD

the expression of E. multilocularis sert and tph (1.03 and 1.16 relative expression compared to untreated controls, respectively; see Additional file 3: Figure S2) suggesting that treatment with paroxetine has no or only a slight effect on the expression of E. multilocularis sert and tph.

Taken together, these results indicate that serotonin transport is crucial for the integrity of E. multilocularis metacestode vesicles and the viability of primary cells.

\section{4-Chloro-DL-phenylalanine does not affect structural integrity of metacestodes}

4-Chloro-DL-phenylalanine is a known inhibitor of $\mathrm{TPH}$ [66], which is the rate-limiting enzyme of serotonin synthesis [67]. To study the role of endogenous serotonin synthesis, we applied 4-chloro-DL-phenylalanine to parasite cultures. Even after 21 days of incubation with 4-chloro-DL-phenylalanine at different concentrations (100, 10 and $1 \mu \mathrm{M})$, metacestodes showed no signs of structural damage. Treatment with
4-chloro-DL-phenylalanine for 2 days also did not affect the viability of primary cells (see Additional file 4: Figure S3).

\section{Discussion}

Serotonin is a phylogenetically ancient molecule with various functions. In addition to its role as a neurotransmitter, serotonin regulates many developmental processes across phyla $[19,22,68]$. In a previous work we and others showed that the E. granulosus genome encodes the proteins required for serotonin biosynthesis, transport and sensing, and that homologues are also present in the E. multilocularis genome [4]. In the present study, we sequenced the genes encoding the $E$. multilocularis SERT, which is required for the uptake of serotonin, and the E. multilocularis $\mathrm{TPH}$, which is the rate-limiting enzyme in endogenous serotonin synthesis [67]. Echinococcus multilocularis TPH contains a Biopterin-dependent aromatic amino acid hydroxylase 
domain and shows high homologies to the Schistosoma mansoni and human TPH, indicating conservation of the enzyme in metazoa. Sequence analysis of E. multilocularis SERT revealed a SNF domain, which is a characteristic of sodium-dependent neurotransmitters, and high homologies to S. mansoni and human SERT. As not all binding sites for paroxetine are conserved in the E. multilocularis SERT and the closely related $S$. mansoni SERT has lower binding affinity for paroxetine than human SERT [69], we used high concentrations of paroxetine in our experiments. While off-target effects cannot be excluded, the binding properties of paroxetine together with the observed effects of paroxetine even at the lower concentration of $10 \mu \mathrm{M}$ suggest that the detected effects are specific.

To investigate the localization of cells expressing $E$. multilocularis sert and tph, we performed WMISH on protoscoleces. The positions and frequencies of cells expressing E. multilocularis sert and tph corresponded to the positions and frequencies of serotonin-containing neurons, as described previously [5], suggesting that both genes are expressed in the nervous system of the protoscolex. A further method to verify this would be co-localization studies using a specific marker for the E. multilocularis nervous system; however, at the present time there are no methods to perform such a study. Similar to our findings, WMISH and immunofluorescence analysis of the planarian Dugesia japonica show expression of the TPH in the planarian nervous system [70]. In addition, available transcriptome data show high expression levels of E. multilocularis sert and tph in non-activated and activated protoscoleces, which could be attributed to expression in the nervous system of the protoscolex. In comparison, the expression of $E$. multilocularis sert and tph in adults is surprisingly low considering the complexity of the serotonergic nervous system described for adults of the closely related species $E$. granulosus $[4,14]$. The relatively high expression of E. multilocularis sert and tph in primary cells could be explained by the presence of nerve cells in primary cell preparations [55]. Likewise, the absence of a serotonergic nervous system at the metacestode stage [5] could account for the low expression levels of E. multilocularis sert and tph in metacestodes. Also, we were not able to detect a specific signal in the WMISH of metacestodes for either gene, which could be due to expression in only a few cells or very low expression in many cells. Taken together, the expression profiles of $E$. multilocularis sert and tph indicate the expression of both genes in nerve cells.

As noted above, serotonin regulates many developmental processes across phyla $[19,22,68]$. Our results indicate that serotonin stimulates the development of
E. multilocularis metacestode vesicles from primary cell preparations and induces proliferation in metacestodes. Serotonin could therefore be an important developmental signal during the formation and growth of the metacestode in the liver. Considering that basal serotonin levels in human blood plasma [20] and in various tissues [71] are lower than $100 \mathrm{nM}$, we observed developmental effects at quite high concentrations. However, serotonin concentrations can reach higher levels during inflammation [71]. Other possibilities are increased serotonin concentrations through endogenous serotonin synthesis or uptake of exogenous serotonin.

The authors of previous studies have proposed that cellular uptake is required for certain mitogenic and developmental effects of serotonin [20, 72, 73]. Serotonin transport is known to be involved in developmental processes in flatworms, such as during the traumatic regeneration of the planarian Polycelis tenuis [27] and the miracidial transformation of S. mansoni $[28,29]$. We have previously reported that exogenous serotonin induces re-differentiation of E. granulosus protoscoleces towards the metacestode stage and that this process is inhibited by citalopram [4]. Here, we have shown that the SSRI paroxetine significantly reduced the viability of primary cells and affected the structural integrity of metacestode vesicles, indicating that serotonin transport is essential for the parasite. Since primary cell cultures are highly enriched in stem cells [55], we expect that paroxetine has not only toxic effects on primary cells but also on stem cells in metacestodes, which might account for the loss of structural integrity of paroxetine-treated metacestodes. While it cannot be ruled out that paroxetine treatment affects the expression of E. multilocularis sert and tph, we found no evidence in that regard, suggesting that the effects of paroxetine cannot be compensated for by the upregulation of serotonin transport or endogenous serotonin synthesis.

Metacestodes lack a serotonergic nervous system [5]. Since, according to transcriptome information reported here, E. multilocularis sert was expressed in metacestodes and the SSRI paroxetine showed clear effects on metacestodes, it is probable that E. multilocularis SERT is not exclusively expressed in nerve cells but also in other cell types. We therefore hypothesize that E. multilocularis SERT fulfills an additional and essential function outside the nervous system. This hypothesis is in accordance with previous results on the $S$. mansoni SERT where the authors propose a predominantly neuronal function for the SERT with a possible secondary role in exogenous serotonin uptake [74]. In addition to a potential role in serotonin uptake from the host, the E. multilocularis SERT might transport serotonin into 
cells where it could act as an intracellular regulator of cell activity, an evolutionary early role of serotonin [19]. In contrast to E. multilocularis sert, expression of the $E$. multilocularis tph appears to be limited to nerve cells. Neither transcriptome information, WMISH nor cell culture experiments provided any indication of a role outside the nervous system. While it cannot be ruled out that the lack of response to 4-chloro-DL-phenylalanine in cell culture experiments is due to 4-chloro-DL-phenylalanine not inhibiting E. multilocularis TPH, this could also be explained by a lack of (nerve) cells that express TPH.

\section{Conclusions}

Serotonin is a widely distributed neurotransmitter and mitogen. Our study provides evidence for both functions in E. multilocularis. Transcriptome data and WMISH suggest that the E. multilocularis tph and sert are expressed in the nervous system of the protoscolex. While the role of the E. multilocularis TPH appears to be restricted to the nervous system, our data indicate that the E. multilocularis SERT has an additional role outside the nervous system and is essential for parasite development and survival. Our data further suggest that serotonin plays an important role in E. multilocularis metacestode development and proliferation. Serotonin could therefore be a contributing factor to the formation and growth of the parasite in the liver.

\section{Supplementary Information}

The online version contains supplementary material available at https://doi. org/10.1186/s13071-020-04533-0.

Additional file 1: Table S1. Primer combinations used for amplification of E. multilocularis sert and E. multilocularis tph.

Additional file 2: Figure S1. Genomic location of E. multilocularis tph.

Additional file 3: Figure S2. Effect of paroxetine on expression of $E$. multilocularis sert and tph.

Additional file 4: Figure S3. Effect of 4-chloro-DL-phenylalanine on E. multilocularis.

\section{Abbreviations}

Biopterin_H: Biopterin-dependent aromatic amino acid hydroxylase; EdU: 5-Ethynyl-2'-deoxyuridine; RACE: Rapid amplification of cDNA ends; RT-PCR: Reverse transcription PCR; SERT: Serotonin reuptake transporter; SNF: Sodium neurotransmitter symporter family; SSRI: Selective serotonin reuptake inhibitor; TPH: Tryptophan hydroxylase; WMISH: Whole mount in situ hybridization.

\section{Acknowledgements}

We thank Federico Camicia, of the "Instituto de Investigaciones en Microbiología y Parasitología Médica", for insightful discussions about serotonin inhibitors.

\section{Authors' contributions}

$\mathrm{MH}$ carried out the experiments and analyzed and interpreted the data. KB made significant contributions to the experimental design and data evaluation. Both authors contributed to the manuscript and approved the submitted version. Both authors read and approved the final manuscript.

\section{Funding}

This work was supported by the Wellcome Trust (https://wellcome.ac.uk/) Grant 107475/Z/15/Z (to KB; FUGI). MH was supported by a grant of the German Excellence initiative to the Graduate School of Life Sciences (GSLS), University of Würzburg. The funding sources had no involvement in any aspect of the scientific work and the publication process.

\section{Availability of data and materials}

The sequences of E. multilocularis sert and tph are available in the EMBL Nucleotide Sequence Database under the accession numbers LT934126.1 (E. multilocularis sert) and LT934127.1 (E. multilocularis tph), https://www.ebi. ac.uk/.

\section{Ethics approval and consent to participate}

Animal experiments were performed according to German and European regulations on animal protection (Tierschutzgesetz) with ethical approval from the local ethics committee of the Government of Lower Franconia (Permit No. 55.2-2531.01/61/13).

\section{Consent for publication}

Not applicable.

\section{Competing interests}

The authors declare that they have no competing interests.

Received: 15 July 2020 Accepted: 10 December 2020

Published online: 06 January 2021

\section{References}

1. Eckert J, Deplazes P. Biological, epidemiological, and clinical aspects of echinococcosis, a zoonosis of increasing concern. Clin Microbiol Rev. 2004; 17:107-35.

2. Dixon JB. Echinococcosis. Comp Immunol Microbiol Infect Dis. 1997;20:87-94.

3. Smyth JD, McManus DP. The physiology and biochemistry of cestodes. Cambridge: Cambridge University Press; 1989.

4. Camicia F, Herz M, Prada LC, Kamenetzky L, Simonetta SH, Cucher MA, et al. The nervous and prenervous roles of serotonin in Echinococcus spp. Int J Parasitol. 2013;43:647-59.

5. Koziol U, Krohne G, Brehm K. Anatomy and development of the larval nervous system in Echinococcus multilocularis. Front Zool. 2013;10:24.

6. McKay DM, Fairweather I, Johnston CF, Shaw C, Halton DW. Immunocytochemical and radioimmunometrical demonstration of serotonin- and neuropeptide-immunoreactivities in the adult rat tapeworm, Hymenolepis diminuta (Cestoda, Cyclophyllidea). Parasitology. 1991;103:275-89.

7. Fairweather I, Macartney GA, Johnston CF, Halton DW, Buchnan KD. Immunocytochemical demonstration of 5-hydroxytryptamine (serotonin) and vertebrate neuropeptides in the nervous system of excysted cysticercoid larvae of the rat tapeworm, Hymenolepis diminuta (Cestoda, Cyclophyllidea). Parasitol Res. 1988;74:371-9.

8. Terenina NB, Poddubnaya LG, Tolstenkov OO, Gustafsson MK. An immunocytochemical, histochemical and ultrastructural study of the nervous system of the tapeworm Cyathocephalus truncatus (Cestoda, Spathebothriidea). Parasitol Res. 2009;104:267-75.

9. Gustafsson MK, Fagerholm HP, Halton DW, Hanzelova V, Maule AG, Reuter M, et al. Neuropeptides and serotonin in the cestode, Proteocephalus exiguus: an immunocytochemical study. Int J Parasitol. 1995;25:673-82.

10. Gustafsson MK, Wikgren MC, Karhi TJ, Schot LP. Immunocytochemical demonstration of neuropeptides and serotonin in the tapeworm Diphyllobothrium dendriticum. Cell Tissue Res. 1985;240:255-60.

11. Fairweather I, Maule AG, Mitchell SH, Johnston CF, Halton DW. Immunocytochemical demonstration of 5-hydroxytryptamine (serotonin) in the nervous system of the liver fluke, Fasciola hepatica (Trematoda, Digenea). Parasitol Res. 1987;73:255-8.

12. Gustafsson MK. Immunocytochemical demonstration of neuropeptides and serotonin in the nervous systems of adult Schistosoma mansoni. Parasitol Res. 1987;74:168-74. 
13. Fairweather I, McMullan MT, Johnston CF, Rogan MT, Hanna RE. Serotoninergic and peptidergic nerve elements in the protoscolex of Echinococcus granulosus (Cestoda, Cyclophyllidea). Parasitol Res. 1994;80:649-56.

14. Brownlee DJ, Fairweather I, Johnston CF, Rogan MT. Immunocytochemical localization of serotonin (5-HT) in the nervous system of the hydatid organism, Echinococcus granulosus (Cestoda, Cyclophyllidea). Parasitology. 1994;109:233-41.

15. Day TA, Bennett JL, Pax RA. Serotonin and its requirement for maintenance of contractility in muscle fibres isolated from Schistosoma mansoni. Parasitology. 1994;108:425-32.

16. Boyle JP, Zaide JV, Yoshino TP. Schistosoma mansoni: effects of serotonin and serotonin receptor antagonists on motility and length of primary sporocysts in vitro. Exp Parasitol. 2000;94:217-26.

17. Sukhdeo MV, Hsu SC, Thompson CS, Mettrick DF. Hymenolepis diminuta: behavioral effects of 5-hydroxytryptamine, acetylcholine, histamine and somatostatin. J Parasitol. 1984;70:682-8.

18. Camicia F, Celentano AM, Johns ME, Chan JD, Maldonado L, Vaca H, et al. Unique pharmacological properties of serotoninergic G-protein coupled receptors from cestodes. PLoS Negl Trop Dis. 2018;12:e0006267.

19. Turlejski K. Evolutionary ancient roles of serotonin: long-lasting regulation of activity and development. Acta Neurobiol Exp. 1996;56:619-36.

20. Fanburg BL, Lee SL. A new role for an old molecule: serotonin as a mitogen. Am J Physiol. 1997;272:L795-806.

21. Gaspar P, Cases O, Maroteaux L. The developmental role of serotonin: news from mouse molecular genetics. Nature Rev Neurosci. 2003:4:1002-12.

22. Lauder JM. Neurotransmitters as growth regulatory signals: role of receptors and second messengers. Trends Neurosci. 1993;16:233-40.

23. Renaud F, Parisi E, Capasso A, De Prisco P. On the role of serotonin and 5 -methoxy-tryptamine in the regulation of cell division in sea urchin eggs. Dev Biol. 1983;98:37-46.

24. Sarrouilhe D, Clarhaut J, Defamie N, Mesnil M. Serotonin and cancer: what is the link? Curr Mol Med. 2015;15:62-77.

25. Seuwen K, Pouyssegur J. Serotonin as a growth factor. Biochem Pharmacol. 1990;39:985-90.

26. Whitaker-Azmitia PM, Druse M, Walker P, Lauder JM. Serotonin as a developmental signal. Behav Brain Res. 1996;73(1-2):19-29.

27. Franquinet $R$. The role of serotonin and catecholamines in the regeneration of the Planaria Polycelis tenvis. J Embryol Exp Morphol. 1979;51:85-95.

28. Taft AS, Norante FA, Yoshino TP. The identification of inhibitors of Schistosoma mansoni miracidial transformation by incorporating a mediumthroughput small-molecule screen. Exp Parasitol. 2010;125:84-94.

29. Kawamoto F, Shozawa A, Kumada N, Kojima K. Possible roles of CAMP and $\mathrm{Ca} 2+$ in the regulation of miracidial transformation in Schistosoma mansoni. Parasitol Res. 1989;75:368-74.

30. Spiliotis M, Mizukami C, Oku Y, Kiss F, Brehm K, Gottstein B. Echinococcus multilocularis primary cells: improved isolation, small-scale cultivation and RNA interference. Mol Biochem Parasitol. 2010;174:83-7.

31. Spiliotis M, Lechner S, Tappe D, Scheller C, Krohne G, Brehm K. Transient transfection of Echinococcus multilocularis primary cells and complete in vitro regeneration of metacestode vesicles. Int J Parasitol. 2008;38:1025-39.

32. Brehm K, Spiliotis M. Recent advances in the in vitro cultivation and genetic manipulation of Echinococcus multilocularis metacestodes and germinal cells. Exp Parasitol. 2008;119:506-15.

33. Spiliotis M, Brehm K. Axenic in vitro cultivation of Echinococcus multilocularis metacestode vesicles and the generation of primary cell cultures. Methods Mol Biol. 2009;470:245-62.

34. Brehm K, Kronthaler K, Jura H, Frosch M. Cloning and characterization of beta-tubulin genes from Echinococcus multilocularis. Mol Biochem Parasitol. 2000;107:297-302.

35. Fernández C, Gregory WF, Loke P, Maizels RM. Full-length-enriched cDNA libraries from Echinococcus granulosus contain separate populations of oligo-capped and trans-spliced transcripts and a high level of predicted signal peptide sequences. Mol Biochem Parasitol. 2002;122:171-80.

36. Tsai IJ, Zarowiecki M, Holroyd N, Garciarrubio A, Sanchez-Flores A, Brooks $\mathrm{KL}$, et al. The genomes of four tapeworm species reveal adaptations to parasitism. Nature. 2013;496:57-63.
37. Logan-Klumpler FJ, De Silva N, Boehme U, Rogers MB, Velarde G, McQuilIan JA, et al. GeneDB - an annotation database for pathogens. Nucleic Acids Res. 2012;40:D98-108.

38. GeneDB. https://www.genedb.org/. Accessed 14 Apr 2017.

39. Hubert K, Zavala-Gongora R, Frosch M, Brehm K. Identification and characterization of PDZ-1, a N-ERMAD specific interaction partner of the Echinococcus multilocularis ERM protein Elp. Mol Biochem Parasitol. 2004;134:149-54

40. Hall TA. BioEdit: a user-friendly biological sequence alignment editor and analysis program for Windows 95/98/NT. Nucl Acids Symp Ser. 1999:41:95-8.

41. Ramakers C, Ruijter JM, Deprez RH, Moorman AF. Assumption-free analysis of quantitative real-time polymerase chain reaction (PCR) data. Neurosci Lett. 2003;339:62-6.

42. Ruijter JM, Ramakers C, Hoogaars WM, Karlen Y, Bakker O, van den Hoff MJ, et al. Amplification efficiency: linking baseline and bias in the analysis of quantitative PCR data. Nucleic Acids Res. 2009;37:e45.

43. Pfaffl MW. A new mathematical model for relative quantification in realtime RT-PCR. Nucleic Acids Res. 2001;29(9):e45.

44. fgStatistics. Statistical software for the analysis of experiments of functional genomics; 2012. http://sites.google.com/site/fgStatistics/. Accessed 04 Sept 2018

45. Howe KL, Bolt BJ, Cain S, Chan J, Chen WJ, Davis P, et al. WormBase 2016 : expanding to enable helminth genomic research. Nucleic Acids Res. 2016;44:D774-80

46. Howe KL, Bolt BJ, Shafie M, Kersey P, Berriman M. WormBase ParaSite-a comprehensive resource for helminth genomics. Mol Biochem Parasitol. 2017;215:2-10

47. WormBaseParaSite. https://parasite.wormbase.org. Accessed 20 Jan 2018.

48. Letunic I, Bork P. 20 years of the SMART protein domain annotation resource. Nucleic Acids Res. 2018;46:D493-6.

49. Letunic I, Doerks T, Bork P. SMART: recent updates, new developments and status in 2015. Nucleic Acids Res. 2015;43:D257-60.

50. Edgar RC. MUSCLE: multiple sequence alignment with high accuracy and high throughput. Nucleic Acids Res. 2004;32(5):1792-7.

51. Edgar RC. MUSCLE: a multiple sequence alignment method with reduced time and space complexity. BMC Bioinform. 2004;5:113.

52. WormBaseParaSite. https://parasite.wormbase.org. Accessed 7 July 2016.

53. Kim D, Langmead B, Salzberg SL. HISAT: a fast spliced aligner with low memory requirements. Nat Methods. 2015;12:357-60.

54. Anders S, Pyl PT, Huber W. HTSeq-a Python framework to work with high-throughput sequencing data. Bioinformatics. 2015;31:166-9.

55. Koziol U, Rauschendorfer T, Zanon Rodriguez L, Krohne G, Brehm K. The unique stem cell system of the immortal larva of the human parasite Echinococcus multilocularis. EvoDevo. 2014;5:10.

56. Schindelin J, Arganda-Carreras I, Frise E, Kaynig V, Longair M, Pietzsch T, et al. Fiji: an open-source platform for biological-image analysis. Nat Methods. 2012;9:676-82.

57. Rueden CT, Schindelin J, Hiner MC, DeZonia BE, Walter AE, Arena ET, et al. Image J2: ImageJ for the next generation of scientific image data. BMC Bioinform. 2017;18:529.

58. Larsen MB, Elfving B, Wiborg O. The chicken serotonin transporter discriminates between serotonin-selective reuptake inhibitors. A speciesscanning mutagenesis study. J Biol Chem. 2004;279:42147-56.

59. Henry LK, Field JR, Adkins EM, Parnas ML, Vaughan RA, Zou MF, et al. Tyr95 and Ile-172 in transmembrane segments 1 and 3 of human serotonin transporters interact to establish high affinity recognition of antidepressants. J Biol Chem. 2006;281:2012-23.

60. Mortensen OV, Kristensen AS, Wiborg O. Species-scanning mutagenesis of the serotonin transporter reveals residues essential in selective, highaffinity recognition of antidepressants. J Neurochem. 2001;79:237-47.

61. Andersen J, Taboureau O, Hansen KB, Olsen L, Egebjerg J, Stromgaard K, et al. Location of the antidepressant binding site in the serotonin transporter: importance of Ser-438 in recognition of citalopram and tricyclic antidepressants. J Biol Chem. 2009;284:10276-84.

62. Thorvaldsdóttir H, Robinson JT, Mesirov JP. Integrative genomics viewer (IGV): high-performance genomics data visualization and exploration. Brief Bioinform. 2013;14:178-92.

63. Robinson JT, Thorvaldsdóttir H, Winckler W, Guttman M, Lander ES, Getz $\mathrm{G}$, et al. Integrative genomics viewer. Nat Biotechnol. 2011;29:24-6. 
64. Walther DJ, Bader M. A unique central tryptophan hydroxylase isoform. Biochem Pharmacol. 2003;66:1673-80.

65. Olson PD, Zarowiecki M, Kiss F, Brehm K. Cestode genomics-progress and prospects for advancing basic and applied aspects of flatworm biology. Parasit Immunol. 2012;34:130-50.

66. Bai Y, Wang HM, Liu M, Wang Y, Lian GC, Zhang XH, et al. 4-Chloro-DL-phenylalanine protects against monocrotalineinduced pulmonary vascular remodeling and lung inflammation. Int J Mol Med. 2014;33:373-82.

67. Lovenberg W, Jequier E, Sjoerdsma A. Tryptophan hydroxylation: measurement in pineal gland, brainstem, and carcinoid tumor. Science. 1967;155:217-9.

68. Azmitia EC. Modern views on an ancient chemical: serotonin effects on cell proliferation, maturation, and apoptosis. Brain Res Bull. 2001;56:413-24

69. Fontana AC, Sonders MS, Pereira-Junior OS, Knight M, Javitch JA, Rodrigues $\mathrm{V}$, et al. Two allelic isoforms of the serotonin transporter from Schistosoma mansoni display electrogenic transport and high selectivity for serotonin. Euro J Pharmacol. 2009;616:48-57.

70. Nishimura K, Kitamura Y, Inoue T, Umesono Y, Yoshimoto K, Takeuchi $K$, et al. Identification and distribution of tryptophan hydroxylase
(TPH)-positive neurons in the planarian Dugesia japonica. Neurosci Res. 2007;59:101-6.

71. Mossner R, Lesch KP. Role of serotonin in the immune system and in neuroimmune interactions. Brain Behav Immun. 1998;12:249-71.

72. Tutton PJ, Barkla DH. Influence of inhibitors of serotonin uptake on intestinal epithelium and colorectal carcinomas. Br J Cancer. 1982;46:260-5.

73. Buznikov GA, Lambert HW, Lauder JM. Serotonin and serotonin-like substances as regulators of early embryogenesis and morphogenesis. Cell Tissue Res. 2001:305:177-86.

74. Patocka N, Ribeiro P. The functional role of a serotonin transporter in Schistosoma mansoni elucidated through immunolocalization and RNA interference (RNAi). Mol Biochem Parasitol. 2013;187:32-42.

\section{Publisher's Note}

Springer Nature remains neutral with regard to jurisdictional claims in published maps and institutional affiliations.
Ready to submit your research? Choose BMC and benefit from:

- fast, convenient online submission

- thorough peer review by experienced researchers in your field

- rapid publication on acceptance

- support for research data, including large and complex data types

- gold Open Access which fosters wider collaboration and increased citations

- maximum visibility for your research: over $100 \mathrm{M}$ website views per year

At BMC, research is always in progress.

Learn more biomedcentral.com/submissions 